\title{
Lung Features in Individuals with Biomass Smoke Exposure Characterized by CT Scan and Changes in Pulmonary Function
}

\author{
Jinglong Chen, ${ }^{1,2, *}$ \\ Changbin Jiang, ${ }^{1}, *$ \\ Youlan Zheng, ${ }^{1} *$ \\ Dongxing Zhao, $\mathbb{D}^{\mathrm{I}, *}$
}

Fan Wu, (D)' Zhuxiang Zhao, ${ }^{3}$

Jun Zhao, ${ }^{2}$ Qing Li, ${ }^{2}$ Bing Li, ${ }^{4}$

Gongyong Peng, (D)

Yumin Zhou,' Pixin Ran (1)

'National Center for Respiratory Medicine, State Key Laboratory of Respiratory Disease, National Clinical

Research Center for Respiratory

Disease, Guangzhou Institute of

Respiratory Health, The First Affiliated

Hospital of Guangzhou Medical

University, Guangzhou, 5I0I20, People's

Republic of China; ${ }^{2}$ Department of

Geriatrics, National Clinical Key

Specialty, Guangzhou First People's

Hospital, South China University of

Technology, Guangzhou, 510180, People's

Republic of China; ${ }^{3}$ The Pulmonary

Medicine, Guangzhou First People's

Hospital, School of Medicine, South

China University of Technology,

Guangzhou, 510180, People's Republic of

China; ${ }^{4}$ GMU-GIBH Joint School of Life

Sciences, Guangzhou Medical University,

Guangzhou, 5 I I436, People's Republic of

China

*These authors contributed equally to this work

Correspondence: Pixin Ran

National Center for Respiratory

Medicine, State Key Laboratory of

Respiratory Disease, National Clinical

Research Center for Respiratory Disease,

Guangzhou Institute of Respiratory

Health, The First Affiliated Hospital of

Guangzhou Medical University,

Guangzhou, 510120, People's Republic of

China

Email pxran@gzhmu.edu.cn
Background and Objective: To determine the effects of BSE (biomass smoke exposure) on pulmonary and non-pulmonary changes in patients with COPD compared with normal individuals.

Methods: Using a cohort, we recruited 16 healthy individuals with BSE (BSE normal), 19 patients with BSE+COPD, 13 healthy individuals with cigarette smoke exposure (CSE normal), 25 patients with CSE $+\mathrm{COPD}$, and 25 healthy controls. Patients with GOLD stage I and II COPD were included. Baseline data (demographic data, BSE or CSE, lung function, and CT findings) and follow-up lung function data were collected. CT parameters of emphysema, pulmonary small vessels, airway remodeling, pectoralis muscles, and erector spinae muscle were measured.

Results: Individuals with BSE were mainly women (32/35, 91.43\%). Compared with the $\mathrm{CSE}+\mathrm{COPD}$ group, the BSE $+\mathrm{COPD}$ group demonstrated slower lung function decline, increased lower lung emphysema, narrower airway lumen dimensions and increased airway wall thickening in the moderate and small airways (all $P<0.05$ ). Compared with healthy controls, the CSE normal and BSE normal groups exhibited significant reductions in pulmonary small vessel area and obvious airway remodeling in small airways $(P<0.05)$. Compared with the BSE normal group, the BSE + COPD group showed significantly more severe emphysema and airway remodeling, as well as reduced left pectoralis major muscle area (all $P<0.05$ ).

Conclusion: Healthy individuals with BSE had reduced pulmonary small vessel area and evidence of airway remodeling; patients with BSE and COPD showed more severe emphysema, airway remodeling, and reductions in pectoralis major muscle area.

Clinical Trial Registration: ChiCTR-OO-14004264.

Keywords: biomass smoke, chronic obstructive pulmonary disease, computed tomography, pulmonary function

\section{Introduction}

Chronic obstructive pulmonary disease (COPD) is associated with exposure to toxic particles or gases. Biomass smoke exposure (BSE) and cigarette smoke exposure (CSE) are the most important risk factors for development of COPD. ${ }^{1,2}$ In recent decades, considerable progress has been made in the treatment of COPD, but its incidence and mortality remain high, which suggests that COPD-related environmental and occupational factors require further attention. ${ }^{1-3}$ Among these factors, the absence of interventions for BSE is particularly obvious. ${ }^{4}$ Nearly 3 billion people worldwide 
use biomass as energy for heating and cooking, which results in substantial BSE exposure. ${ }^{1}$ Individuals affected by BSE mainly women who use biomass for cooking in underdeveloped rural areas; these individuals have relatively lower socioeconomic status. ${ }^{5-8}$

BSE has received insufficient attention..$^{9-11}$ For example, among users of Douyin (a popular social media service in China), there are currently 198 accounts that promote smoking cessation-related content; by contrast, only one professional account addresses the risks of biomass smoke pollution. BSE COPD patients have specific miRNA profile and pathological changes, such as more bronchiolitis, pulmonary fibrosis, and airway wall thickness. Moreover, few studies have reported clinical symptoms and the computed tomography (CT) findings of emphysema and/or small airway disease caused by BSE. They are presented with lower oxygen saturation and weaker activity tolerance compared with CSE COPD patients. ${ }^{12-16}$ There is a lack of comprehensive studies regarding pulmonary lesions caused by BSE, how BSE affects the pulmonary small vessels, the respiratory muscles, the development of emphysema and airway remodeling.

Our study focused on populations with BSE in economically underdeveloped rural areas to comprehensively investigate $\mathrm{CT}$ findings in patients with and without COPD and exposures to cigarette smoke or biomass fuels. This study aimed to clarify the features of BSE-related lung injury compared to CSE induced injury that results in COPD vs those without COPD.

\section{Methods}

\section{Study Design}

The Ethics Committee of Guangzhou Institute of Respiratory Health approved the study protocol. This study was conducted in accordance with the Declaration of Helsinki. Participants were selected from among the rural population in an underdeveloped mountainous area of northern Guangdong, China, using an observatory research subgroup data from COPD community screening database (from 2014 to 2015). ${ }^{13,17,18}$ No intervention was performed on participants and no medication was prescribed to patients with COPD. BSE exposure population and CSE population were followed for a year. Healthy control individuals were not followed up with pulmonary function. Written informed consent was obtained from all participants. Detailed information regarding recruitment, clinical management, and follow-up was described in previous studies. ${ }^{13,17,18}$

\section{Population}

This study included individuals with BSE or CSE (including healthy individuals and patients with COPD) and healthy controls, who were $>40$ years of age, had complete clinical data, pulmonary function assessments, and lung HRCT data. The study excluded patients who had lung cancer, asthma, interstitial lung disease, pulmonary infarction, pneumonia, or pleural effusion, based on medical history or CT scan findings. The healthy control group included healthy individuals with normal lung function, without a history of CSE or BSE, without a history of pulmonary disease or respiratory symptoms, and without visible emphysema or low attenuation areas less than a threshold of -950 Hounsfield units (LAA-950\%) $<5 \%$ in CT scans.

\section{Patient Groups}

According to their history of CSE or BSE and whether they had been diagnosed with COPD, 98 eligible participants were allocated into five groups: BSE + COPD $(n=19)$, BSE normal $(n=16), C S E+C O P D(n=25)$, CSE normal $(n=13)$, and healthy control $(n=25)$ (Figure 1). The BSE + COPD and CSE+COPD groups only included patients with Global Initiative for Chronic Obstructive Lung Disease (GOLD) stages I and II (post-bronchodilator forced expiratory volume in 1 second $\left[\mathrm{FEV}_{1}\right]$ /forced vital capacity $[\mathrm{FVC}]<0.7$ and $\mathrm{FEV}_{1} \geq 50 \%$ predicted).

\section{Data Collected}

Baseline records of participants were collected, including demographic information, lung function, history of BSE and/ or CSE, and high-resolution CT findings; follow-up lung function data were also collected. The BSE and CSE indexes were calculated as previously described. ${ }^{13}$ The BSE index is defined as the cumulative exposure of biomass, calculated by multiplying the average number of hours per day in the kitchen with the number of years of cooking with biomass. Cigarette smoking index was expressed as pack-years.

Annual reductions in lung function $\left(\mathrm{FEV}_{1}\right.$ and $\left.\mathrm{FVC}\right)$ were calculated. High-resolution CT findings were assessed to determine the severity of emphysema and airway remodeling, as well as damage to pulmonary small vessels, pectoralis major muscle, pectoralis minor muscle, and erector spinae muscle.

\section{Imaging Methods}

Quantitative CT Imaging Analysis

High-resolution CT was performed at suspended full inspiration using a multidetector row CT scanner (Aquilion 16, 


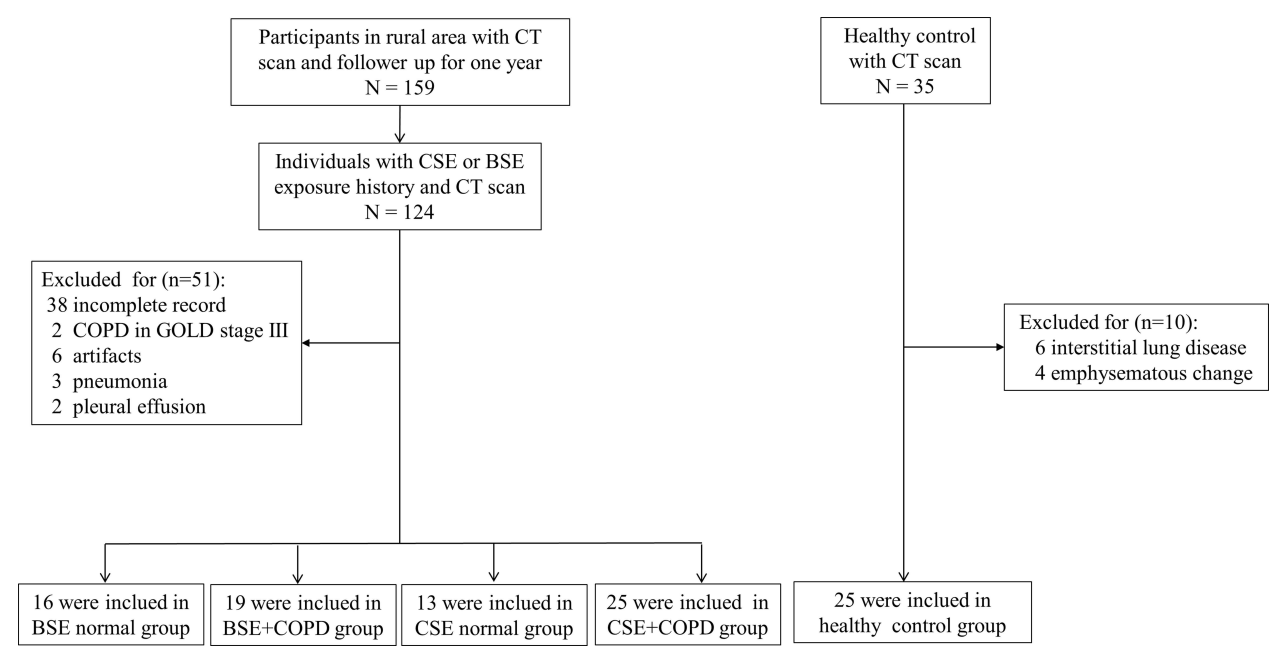

Figure I Flow chart of the study participants.

Abbreviations: CT, computed tomography; CSE, cigarette smoke exposure; BSE, biomass smoke exposure; COPD, chronic obstructive pulmonary disease; GOLD, Global Initiative for Chronic Obstructive Lung Disease.

Toshiba, Tokyo, Japan). ${ }^{13} \mathrm{CT}$ scan findings were analyzed with 3D slicer software, version 4.8.1 (https://www.slicer. org, Brigham and Women's Hospital).

\section{CT Measurement of Emphysema}

Emphysema was detected using the chest imaging platform/parenchyma module of 3D slicer software, using a Hounsfield unit threshold of -950 (ie, \%LAA-950). The (upper third)/(lower third) ratio of LAA-950 was used to assess the distribution of emphysema. ${ }^{19,20}$

\section{CT Measurement of Bronchial Inner Diameter and Bronchial Wall Thickness}

Using the Airway Inspector module of 3D slicer software, the third, fourth, fifth, sixth generation of airway bronchi of the right upper lobe apical bronchus (ie, RB1) and right lower lobe posterior bronchus (ie, RB10) were identified. ${ }^{21}$ Then, the inner diameter, bronchial wall thickness, and bronchial wall area $\%$ were detected.

\section{CT Measurement of Cross-Sectional Area (CSEA)\% of Pulmonary Small Vessels}

In accordance with the method described by Matsuoka et al, pulmonary small vessels were defined as "circular" blood vessels that were perpendicular to the crosssectional plane and exhibited a cross-sectional area of $<5 \mathrm{~mm}^{2}{ }^{21,22}$ The upper, middle, and lower slices were captured $1 \mathrm{~cm}$ above the top of the aortic arch, $1 \mathrm{~cm}$ below the carina, and $1 \mathrm{~cm}$ below the right lower pulmonary vein, respectively. $\mathrm{CSEA}_{<5 \%}$ was calculated as the percentage of small vessel CSEA to lung CSEA in each slice.
The total $\operatorname{CSEA}_{<5 \%}$ was calculated as the percentage of total small vessel CSEA to total lung CSEA of three slices.

CSEA\% detection was performed as shown in Figure 2. First, the segmentation and modeling of the lungs were conducted using the Parenchyma Analysis and edit modules in the 3D slicer software. The segmented lung areas were filled with -200 to achieve a uniformly gray color. Second, chest vessels were segmented using the VTMK module in 3D slicer software. Pulmonary vessels were segmented by clipping chest vessels with the lung model. Third, in the above three slices, images of pulmonary vessels and gray lungs were saved separately. Image-Pro Plus 6.0 software (Media Cybernetics, Inc.) was used to measure the areas of gray lungs and small pulmonary vessels. Fourth, pulmonary vessels with "Rounders" of $0.9-1$ (ie, circular blood vessels) and "Areas" of $\leq 5 \mathrm{~mm}^{2}$ were segmented as pulmonary small vessels $\left(\mathrm{CSEA}_{<5}\right)$. Fifth, CSEAs of gray lungs and small vessels were measured using the count/size module in Image-Pro Plus software for each slice. Finally, $\mathrm{CSEA}_{<5 \%}$ in each slice and total $\mathrm{CSEA}_{<5 \%}$ were calculated using the formula described above.

\section{CT Measurement of Pectoralis and Erector Spinae Muscles}

The area and density of muscles were measured using the body composition module of 3D slicer software, as shown in Figure 3. The pectoralis major and minor muscles were measured at the first slice above the aorta, while the erector spinae muscle was measured at the lower slice of the T12 thoracic vertebra. ${ }^{23,24}$ 
A

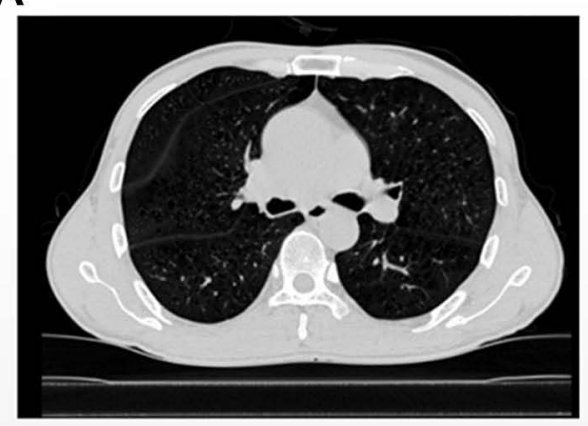

C

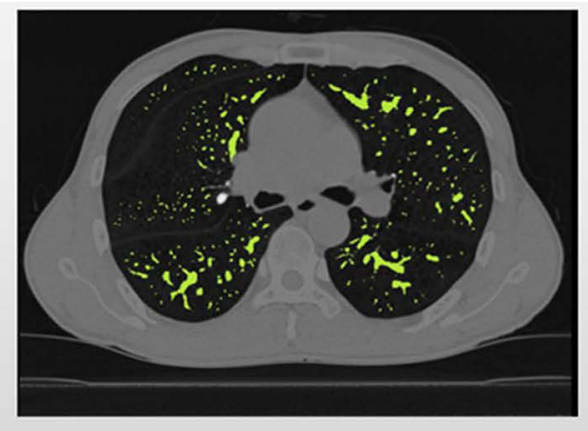

B

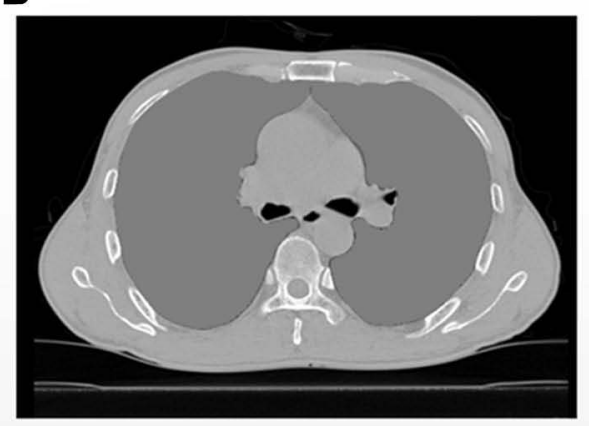

D

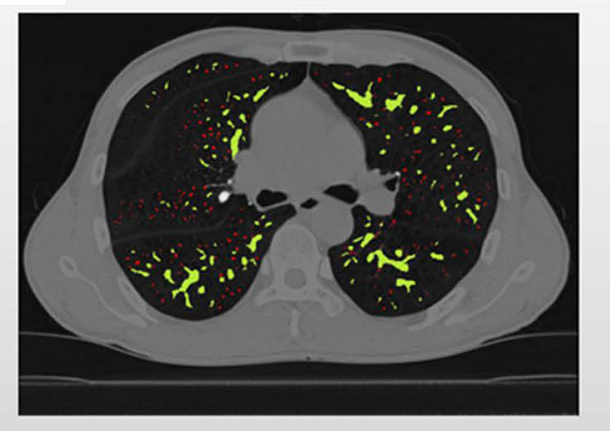

Figure 2 Sample computed tomography (CT) scans used to determine pulmonary small vessels. (A) CT image in middle slice of lung. (B) Segmented lungs shaded in grey. (C) Pulmonary vessels shaded in green. (D) Pulmonary small vessels shaded in red.

A

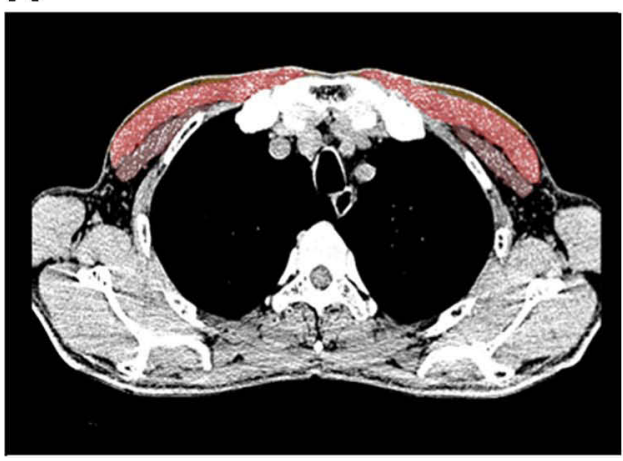

B

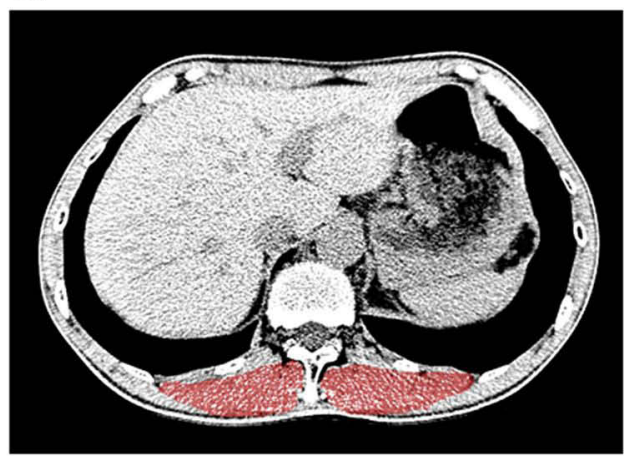

Figure 3 Sample computed tomography (CT) scans used to determine pectoralis muscle, erector spinae muscle. (A) Pectoralis major muscle shaded in red, pectoralis minor muscle shaded in brown. (B) Erector spinae muscle shaded in red.

\section{Statistical Analysis}

Data are presented as mean \pm standard deviation, median (quartile), or numbers. Comparisons of continuous variables among groups or between multiple groups were evaluated by one-way analysis of variance with a post hoc test (least significant difference method), KruskalWallis test or Mann-Whitney $U$-test. Comparisons of categorical variables among groups were performed using Fisher's exact test. Pearson's correlation coefficients were used to assess associations between lung function decline and CT index. Statistical analyses were conducted using IBM SPSS Statistics, version 23.0 (IBM Corp., Armonk, NY, USA). Two-tailed $P$-values $<0.05$ were considered statistically significant.

\section{Results}

\section{Baseline Characteristics}

Table 1 describes the baseline characteristics of participants in each group. This study focused on economically underdeveloped mountainous rural areas, where individuals with BSE 
Table I Baseline Characteristics Among BSE, CSE Groups and Healthy Control Groups

\begin{tabular}{|c|c|c|c|c|c|c|}
\hline & \multicolumn{2}{|c|}{ BSE Groups } & \multicolumn{2}{|c|}{ CSE Groups } & \multirow[t]{2}{*}{ Healthy Control } & \multirow[t]{3}{*}{$P$} \\
\hline & BSE Normal & BSE+ COPD & CSE Normal & CSE+ COPD & & \\
\hline & $n=16$ & $n=19$ & $n=13$ & $n=25$ & $n=25$ & \\
\hline Sex & & & & & & $<0.01^{\mathrm{a}}$ \\
\hline Male & 2 & 1 & 13 & 25 & 16 & \\
\hline Female & 14 & 18 & 0 & 0 & 9 & \\
\hline Age, years & $59(57-66)$ & $67(57-72)$ & $63(59-67)$ & $65(60-70)$ & $61(58-70)$ & $0.19^{\mathrm{a}}$ \\
\hline BMI, $\mathrm{kg} / \mathrm{m}^{2}$ & $22.38 \pm 3.27$ & $23.78 \pm 5.39$ & $23.68 \pm 3.34$ & $21.6 \pm 3.67$ & $24.2 \pm 3.69$ & $0.14^{\mathrm{a}}$ \\
\hline $\mathrm{FVC} \%$ & $101.3(95.0-111.4)$ & $124.5(113.1-136.0)$ & $102.9(102.5-111.8)$ & $106.0(103.3-112.9)$ & $99.6(94.3-105.0)$ & $0.03^{\mathrm{a}}$ \\
\hline $\mathrm{FEV}_{1} \%$ & $95.0(87.8-100.0)$ & $100.5(87.9-113.1)^{\dagger}$ & $96.8(93.6-102.5)$ & $87.7(80.1-89.7)^{* \dagger}$ & $83.8(82.3,94.9)$ & $<0.01^{\mathrm{a}}$ \\
\hline $\mathrm{FEV}_{\mathrm{I}} / \mathrm{FVC}$ & $84.0(76.1-86.2)$ & $66.2(63.5-68.8)^{* \dagger}$ & $77.4(75.8-82.9)$ & $63.8(60.5-66.5)^{* \dagger}$ & $94.2(87.6-95.1)$ & $<0.01^{\mathrm{a}}$ \\
\hline \multicolumn{7}{|l|}{ GOLD stage, $n$} \\
\hline 1 & NA & 13 & NA & 14 & NA & $0.45^{\mathrm{b}}$ \\
\hline ॥ & NA & 6 & NA & 11 & NA & \\
\hline BSE index (hours-years) & $51(37.5,82)$ & $40(20,60)$ & $0(0,0)$ & $0(0,0)$ & $0(0,0)$ & $0.38^{\mathrm{c}}$ \\
\hline Cigarette smoking index (pack-years) & $0(0,0)$ & $0(0,0)$ & $39(20,76)$ & $40(27.5,54)$ & $0(0,0)$ & $0.52^{\mathrm{d}}$ \\
\hline Declined FEV , (mL/year) & $5.1(-10.0$ to 45.5$)$ & $21.4(0 \text { to } 42.7)^{\S}$ & 40.0 (28.5 to 56.8$)$ & 69.5 (39.8 to II3.5) & NA & $<0.01^{\mathrm{b}}$ \\
\hline Declined FVC (mL/year) & $27.9(-50.8$ to 58.3$)$ & 38.7 (2.75 to 88.8$)$ & 31.1 (17.8 to 54.9$)$ & $62.9(32.1$ to 112.2$)$ & NA & $0.48^{\mathrm{b}}$ \\
\hline Declined FEV $/$ /FVC (\%/year) & $0.19(-0.52$ to 1.28$)$ & $-0.32(-1.1 \text { to } 0.47)^{\S}$ & 0.79 (0.45 to 1.08$)$ & $0.89(-0.2$ to 1.56$)$ & NA & $0.03^{\mathrm{b}}$ \\
\hline
\end{tabular}

Notes: Values were given as mean \pm SD or median (quartile). ${ }^{a}$ Comparison among five groups. ${ }^{b}$ Comparison between BSE $+C O P D$ group and CSE $+C O P D$ group. ${ }^{c}$ Comparison between BSE normal group and BSE+COPD group. ${ }^{d}$ Comparison between CSE normal group and CSE+COPD group. *Compared between same subgroup, $P<0.05$. ${ }^{\dagger}$ Compared with healthy control group, $P<0.05$. ${ }^{\S}$ Compared with $C S E+C O P D$ group, $P<0.05$.

Abbreviations: BSE, biomass smoke exposure; CSE, cigarette smoke exposure; FEV applicable.

were mainly women who cook with biofuel, while smokers were mainly men. The BIOFUEL-index for BSE COPD and the smoking index for CSE COPD were not significantly different compared to the BSE and CSE normal groups $(P>0.05)$. There were no significant differences in baseline lung function $\left(\mathrm{FEV}_{1} \%\right.$ and $\left.\mathrm{FEV}_{1} / \mathrm{FVC}\right)$ and $\mathrm{GOLD}$ stage between the BSE+COPD and CSE+COPD groups (both $P>0.05$ ).

This study discovered that lung function decline $\left(\mathrm{FEV}_{1}\right.$ and $\mathrm{FEV}_{1} / \mathrm{FVC}$ ) was slower in the BSE+COPD group than in the CSE + COPD group (both $P<0.05$ ), suggesting that patients with BSE and COPD may exhibit slower progression of disease and may have greater benefit from early active intervention.

\section{CT Features of Individuals with BSE Emphysema Distribution in Patients with BSE and COPD}

Like patients in the CSE+COPD group, those in the BSE + COPD group had greater extent of emphysema than BSE normal group; however, there was no significant difference in the total percentage of emphysema between the two groups $(P>0.05)$. Lower lung emphysema was more in the BSE+COPD group than in the CSE+COPD group $(P<0.05)$. See Table 2.

\section{Pulmonary Small Vessel Area in Healthy Individuals with BSE}

Compared with the healthy control group, both the BSE normal and CSE normal groups showed reduced pulmonary small vessel areas $(P<0.05)$; this was consistent among the upper, middle, and lower regions of the lung $(P<0.05)$. Compared with the BSE normal and CSE normal groups, the pulmonary small vessel areas in BSE+COPD and CSE + COPD groups were further reduced, but these differences were not statistically significant (all $P>0.05$ ). Finally, there was no significant difference in the reduction of pulmonary small blood vessel areas between the BSE+COPD and CSE + COPD groups $(P>0.05)$. See Table 2 and Supplementary Table 1. 
Table 2 Comparison of CT Characteristics on Emphysema, Pulmonary Small Vessel and Airway Remodeling Among BSE, CSE Groups and Healthy Groups

\begin{tabular}{|c|c|c|c|c|c|c|}
\hline & \multicolumn{2}{|c|}{ BSE Groups } & \multicolumn{2}{|c|}{ CSE Groups } & \multirow[t]{2}{*}{ Healthy Control } & \multirow[t]{3}{*}{$P^{a}$} \\
\hline & BSE Normal & BSE+COPD & CSE Normal & CSE+ COPD & & \\
\hline & $n=16$ & $n=19$ & $n=13$ & $n=25$ & $n=25$ & \\
\hline LAA-950 (\%) & $4.17(0.53-5.60)$ & $7.84(5.17-11.36)^{\dagger *}$ & $3.17(1.52-6.00)$ & $|2.6|(5.79-17.36)^{* \dagger}$ & $1.96(1.23-3.4)$. & $<0.01$ \\
\hline $\begin{array}{l}\text { (Upper third)/(lower } \\
\text { third) ratio of LAA-950 }\end{array}$ & $0.96(0.37-1.27)^{\dagger}$ & $0.73(0.54-0.9)^{\S \dagger}$ & $1.00(0.67-1.73)$ & $1.17(0.72-1.75)$ & $0.68(0.65-1.04)$ & 0.01 \\
\hline$\% \mathrm{CSA}_{<5}$ & $1.05(0.84-1.45)^{\dagger}$ & $0.86(0.65-0.98)^{\dagger}$ & $0.93(0.91-0.99)^{\dagger}$ & $1.10(0.66-1.32)^{\dagger}$ & $1.80(1.27-2.14)$ & $<0.01$ \\
\hline $\begin{array}{c}\text { RBI Third } \\
\text { IR (mm) } \\
\text { WA\% }\end{array}$ & $\begin{array}{c}3.47 \pm 0.94 \\
71.57 \pm 7.72^{\dagger}\end{array}$ & $\begin{array}{l}3.24 \pm 0.81^{\dagger \S} \\
71.98 \pm 7.22^{\dagger \S}\end{array}$ & $\begin{array}{l}3.7 I \pm 0.75 \\
65.15 \pm 7.1\end{array}$ & $\begin{array}{c}4.2 \pm 0.87 \\
66.04 \pm 7.58\end{array}$ & $\begin{array}{c}3.85 \pm 1.12 \\
61.81 \pm 8.73\end{array}$ & $\begin{array}{l}0.02 \\
<0.01\end{array}$ \\
\hline $\begin{array}{l}\text { RBI Fourth } \\
\text { IR (mm) } \\
\text { WA\% }\end{array}$ & $\begin{array}{c}2.11(1.86-2.41) \\
78.11(74.17-80.64)^{\dagger}\end{array}$ & $\begin{array}{c}2.09(1.76-2.52) \\
77.12(75.07-81.6)^{\dagger \S}\end{array}$ & $\begin{array}{c}2.48(2.39-2.8) \\
75.00(68.03-77.25)\end{array}$ & $\begin{array}{c}2.42(2.08-2.7) \\
72.05(69.87-76.27)\end{array}$ & $\begin{array}{c}2.47(1.97-2.95) \\
69.8(66.16-72.98)\end{array}$ & $\begin{array}{l}0.06 \\
<0.01\end{array}$ \\
\hline $\begin{array}{l}\text { RBI Fifth } \\
\text { IR (mm) } \\
\text { WA\% }\end{array}$ & $\begin{array}{c}1.65 \pm 0.38^{\dagger} \\
82.76 \pm 5.82^{\dagger}\end{array}$ & $\begin{array}{c}1.66 \pm 0.5^{\dagger \S} \\
80.85 \pm 4.56^{\dagger}\end{array}$ & $\begin{array}{c}1.99 \pm 0.46 \\
78.39 \pm 5.43^{\dagger}\end{array}$ & $\begin{array}{c}2.01 \pm 0.39 \\
78.21 \pm 4.85^{\dagger}\end{array}$ & $\begin{array}{c}1.95 \pm 0.48 \\
72.26 \pm 6.74\end{array}$ & $\begin{array}{l}0.05 \\
<0.01\end{array}$ \\
\hline $\begin{array}{c}\text { RBI Sixth } \\
\text { IR (mm) } \\
\text { WA\% }\end{array}$ & $\begin{array}{c}1.27(0.88-1.6)^{\dagger} \\
84.29(80.92-90.88)^{\dagger}\end{array}$ & $\begin{array}{c}1.16(0.88-1.40)^{\dagger \S} \\
87.73(85.57,90.88)^{\dagger \S}\end{array}$ & $\begin{array}{c}1.53(1.28-1.58) \\
82.44(82.02-88.65)^{\dagger}\end{array}$ & $\begin{array}{c}1.44(1.24-1.64) \\
82.24(75.98-86.44)^{\dagger}\end{array}$ & $\begin{array}{c}1.47(1.33-1.80) \\
76.86(71.72-79.03)\end{array}$ & $\begin{array}{c}0.01 \\
<0.01\end{array}$ \\
\hline $\begin{array}{l}\text { RBI0 Third } \\
\text { IR (mm) } \\
\text { WA\% }\end{array}$ & $\begin{array}{c}3.25 \pm 0.7 I^{\prime} \\
70.82 \pm 6.8 I^{\dagger}\end{array}$ & $\begin{array}{l}3.07 \pm 0.73^{\dagger \S} \\
71.79 \pm 6.77^{\dagger}\end{array}$ & $\begin{array}{c}3.95 \pm 0.59 \\
65.21 \pm 5.69^{\dagger}\end{array}$ & $\begin{array}{c}3.63 \pm 0.58 \\
67.56 \pm 6.08^{\dagger}\end{array}$ & $\begin{array}{l}3.58 \pm 0.62 \\
62.44 \pm 5.2\end{array}$ & $\begin{array}{c}0.02 \\
<0.01\end{array}$ \\
\hline $\begin{array}{l}\text { RBI0 Fourth } \\
\text { IR (mm) } \\
\text { WA\% }\end{array}$ & $\begin{array}{c}2.60(1.85-3.08)^{\dagger \neq} \\
74.03(68.08-83.46)^{\dagger}\end{array}$ & $\begin{array}{c}1.98(1.81-2.54)^{\dagger \S} \\
76.55(70.18-80.5 I)^{\dagger}\end{array}$ & $\begin{array}{c}3.34(2.33-4.14) \\
65.44(58.76-73.49)\end{array}$ & $\begin{array}{c}2.99(2.5 \mathrm{I}-3.82) \\
72.58(65.72-77.2 \mathrm{I})^{\dagger}\end{array}$ & $\begin{array}{c}2.9 \mid(2.4 I-3.6) \\
65.15(60.7|-7| .44)\end{array}$ & $\begin{array}{r}0.03 \\
<0.01\end{array}$ \\
\hline $\begin{array}{c}\text { RBI0 Fifth } \\
\text { IR (mm) } \\
\text { WA\% }\end{array}$ & $\begin{array}{c}1.84(1.49-2.53)^{\dagger \ddagger} \\
78.11(71.02-81.63)^{\dagger}\end{array}$ & $\begin{array}{c}1.37(1.24-2.6)^{\S \dagger} \\
82(74.61-86.97)^{\dagger \S}\end{array}$ & $\begin{array}{c}2.88(2.4-2.98) \\
73.98(69.65-76.07)\end{array}$ & $\begin{array}{c}2.40(1.85-3) \\
77.40(72.86-81.78)^{\dagger}\end{array}$ & $\begin{array}{c}2.31(2.12-2.81) \\
68.46(65.44-72.6)\end{array}$ & $\begin{array}{l}<0.01 \\
<0.01\end{array}$ \\
\hline $\begin{array}{l}\text { RBI0 Sixth } \\
\text { IR (mm) } \\
\text { WA\% }\end{array}$ & $\begin{array}{c}1.60(1.15-1.99)^{\dagger} \\
83.72(79.38-87.45)^{\dagger}\end{array}$ & $\begin{array}{c}1.11(1-1.55)^{\dagger \S} \\
87.53(84.35-87.53)^{\dagger \S}\end{array}$ & $\begin{array}{c}1.86(1.69-2.48) \\
81.56(74.32-82.87)\end{array}$ & $\begin{array}{c}1.82(1.51-2.25) \\
80.45(75.89-84.26)^{\dagger}\end{array}$ & $\begin{array}{c}2.12(I .7-2.4 I) \\
74.07(72.23-77.96)\end{array}$ & $\begin{array}{l}<0.01 \\
<0.01\end{array}$ \\
\hline
\end{tabular}

Notes: Values were given as mean \pm SD or median (quartile). ${ }^{a}$ Comparison among five groups. *Compared between same subgroup, $P<0.05$. ${ }^{\dagger}$ Compared with healthy control group, $P<0.05$. ${ }^{\ddagger}$ Compared with CSE normal group, $P<0.05$. ${ }^{\S}$ Compared with CSE+COPD group, $P<0.05$.

Abbreviations: \%LAA-950, CT measurement of the percentage of low attenuation area less than -950 Hounsfield units, defined as emphysema; \%CSA $<5$, percentage of total lung area taken up by the cross-sectional area of pulmonary vessels less than $5 \mathrm{~mm}^{2}$; IR, inner radius; RBI, upper lobe apical bronchus; RBI0, right lower lobe posterior bronchus. WA\%, percentage of wall.

\section{Airway Remodeling in Medium and Small Airways (Grades III-VI Bronchi)}

Small airway remodeling was present in otherwise healthy individuals with BSE, while patients with BSE and COPD exhibited serious medium and small airway remodeling. Compared with the CSE normal group, small airway remodeling (ie, narrow airway and thickened airway wall) was obvious in grades IV-VI bronchi in the BSE normal group (all $P<0.05$ ). Compared with the CSE
+ COPD group, more serious airway remodeling in medium and small airways (grades III-VI bronchi) was observed in the BSE+COPD group (all $P<0.05$ ). See Table 2 and Supplementary Table 1.

\section{Left Pectoralis Major Muscle Areas in Patients with BSE and COPD}

The left and right pectoralis major muscle area and density were considerably greater in the BSE normal group than in 
the healthy control group (all $P<0.05$ ); similar findings were present in the CSE normal group (all $P<0.05$ ). Compared with the BSE normal group, the left pectoralis major muscle area significantly decreased in the BSE + COPD group $(P<0.05)$. See Table 3 .

\section{CT Presentation and Lung Function Changes}

Lung function decline was relatively slow in the BSE +COPD group; there were no correlations between CT indexes and annual reduction of $\mathrm{FEV}_{1}$ (all $P>0.05$ ). However, $\mathrm{FEV}_{1}$ reduction was relatively rapid in the CSE+COPD group; this reduction negatively correlated with right pectoralis minor muscle area $(\mathrm{r}=-0.68$, $P<0.01$; See Supplementary Table 2).

\section{Discussion}

The harmfulness of smoking is well known because of social media and smoking cessation clinics. ${ }^{10,11,25,26}$ In contrast, the risks of BSE are not well known among the public and health-related efforts are insufficient.

This study was conducted in an underdeveloped mountainous area of rural China. The composition of the study population was representative of the local region. The study specifically focused on describing the lung damage caused by BSE, with CSE used as a reference exposure.

The limited evidence regarding lung damage caused by BSE has mainly emphasized emphysema and air trapping, which does not provide sufficient insights to guide BSE intervention. ${ }^{12-14}$ In this study, we used 3D slicer software to make an overall assessment of lung damage, including airway remodeling in medium and small airways, emphysema, pulmonary small vessels, and respiratory muscles. Qualitative and quantitative determinations of lung damage caused by BSE were performed following threedimensional and two-dimensional imaging of the lung.

Different from previous reports, we discovered that airway damage was more serious in the BSE+COPD group than in the CSE+COPD group, such that it involved substantial remodeling of small and medium airways. ${ }^{12,13}$ Furthermore, pectoralis major muscle area decreased in the $\mathrm{BSE}+\mathrm{COPD}$ group, compared with the BSE normal group; a corresponding difference was not observed between CSE $+\mathrm{COPD}$ and CSE normal groups. The impacts of BSE on skeletal muscle changes compared with CSE require further investigation.

An interesting finding was that both the BSE+COPD and BSE normal groups had significant reductions in pulmonary small vessel area. To the best of our knowledge, this is the first study to show changes in pulmonary small vessel area with BSE. The reduction of pulmonary small vessel area is associated with acute exacerbation of COPD and serves as an independent risk factor for mortality. ${ }^{27,28}$ Additionally, BSE is associated with elevated risks of hypertension, coronary heart disease, and stroke. ${ }^{29}$

The present study showed that lung function decline was slightly slower in the BSE+COPD group, which was consistent with the results reported by Salvi et al. ${ }^{30}$ Since efforts to improve cooking fuels and kitchen ventilation will reduce indoor $\mathrm{PM}_{2.5}$ concentrations, delay lung function decline, and lower the risk of respiratory disease, early intervention for BSE patients may provide greater clinical improvements. ${ }^{18,31}$

Phenotyping studies regarding COPD have found that specific lung CT features are closely related to the progression of COPD. Specific types of emphysema, airway remodeling, and changes in pulmonary small vessel and chest muscle areas, all of which can be reversed by active intervention, are associated with the progression of COPD. ${ }^{19,21,23,27,28,32-34}$ Therefore, we examined correlations between these pathological changes and the annual reduction of $\mathrm{FEV}_{1}$, with the aim of identifying a target to delay lung function decline in patients with BSE and COPD. However, we did not find an association between any $\mathrm{CT}$ index and reduction of $\mathrm{FEV}_{1}$ in the $\mathrm{BSE}+\mathrm{COPD}$ group. This may have been influenced by the small number of participants in the BSE+COPD group, therefore producing no statistically significant findings.

Age is an important risk factor for COPD. In normal people, alveolar sacs increase in size with age. However, the pathological changes of COPD and aging lung are different. $^{35}$ In this study, BSE+COPD patients have significant airway remodeling changes in the middle and small airways, and relatively mild emphysema, suggesting that this change is mainly caused by biomass exposure rather than aging.

This study had some limitations. First, the number of participants was relatively small, and there were no followup lung CT data for any participants. Second, this study did not specifically recruit women in the CSE+COPD group or men in the BSE+COPD group. Data from COPDGene show that gender differences have an impact on the clinical phenotype and acute exacerbation of COPD. ${ }^{36}$ In current study, the differences in gender proportions exist may also have biased the results. Therefore, future studies should focus on sex differences between $\mathrm{BSE}+\mathrm{COPD}$ and $\mathrm{CSE}+\mathrm{COPD}$ groups and the effect of sex on the phenotype of COPD. 


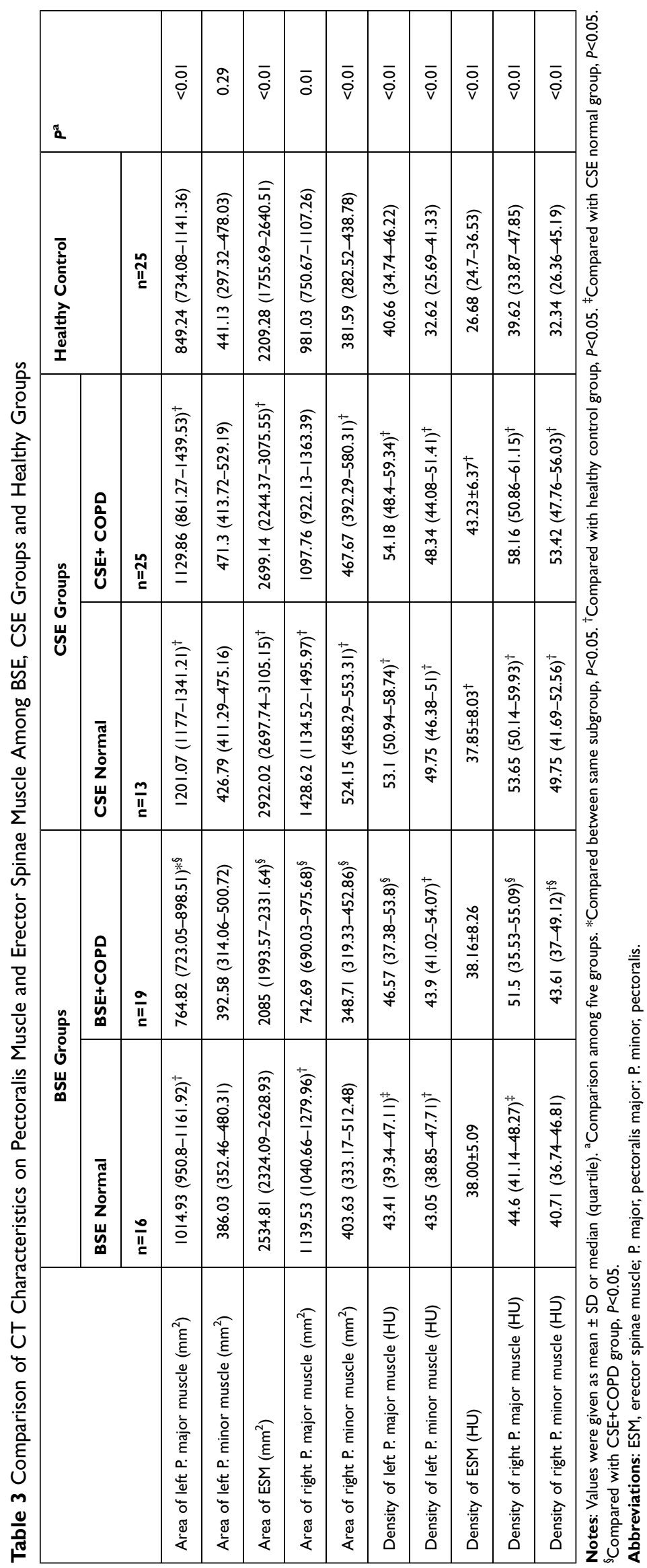


Third, we did not recruit GOLD stage III participants. Fourth, due to insufficient sample size, no comparison was made between groups of GOLD stage I and GOLD stage II for patients with $\mathrm{BSE}+\mathrm{COPD}$, and no comparison between $\mathrm{BSE}$ COPD and CSE COPD patients with the same GOLD stage. Future subgroup study based on GOLD stage will add show more value to the effect of BSE on lung CT findings.

In summary, this study focused on individuals with BSE living in an underdeveloped rural area of China. It showed that otherwise healthy individuals exposed to BSE had reduced pulmonary small vessel area and airway remodeling, while patients with BSE and COPD had more severe emphysema, airway remodeling, and reductions in pectoralis muscle change, as well as relatively slower declines in lung function. More investigations are thus needed regarding the specific phenotypes of BSE COPD patients in different stage of GOLD.

\section{Abbreviations}

BSE, biomass smoke exposure; COPD, chronic obstructive pulmonary disease; CSE, cigarette smoke exposure; CSEA, cross-sectional area; CT, computed tomography; $\mathrm{ESM}$, erector spinae muscle; $\mathrm{FEV}_{1}$, forced expiratory volume in 1 second; FVC, forced vital capacity; GOLD, Global Initiative for Chronic Obstructive Lung Disease; IR, inner radius; P. major, pectoralis major; P. minor, pectoralis minor; RB1, upper lobe apical bronchus; RB10, right lower lobe posterior bronchus; WT, wall thickness; WA\%, percentage of wall; \%LAA-950, CT measurement of the percentage of low attenuation area less than -950 Hounsfield units, defined as emphysema; $\% \mathrm{CSA}<5$, percentage of total lung area taken up by the cross-sectional area of pulmonary vessels less than $5 \mathrm{~mm}^{2}$.

\section{Summary at Glance}

This is the first report on pulmonary and non-pulmonary damage on BSE population by $\mathrm{CT}$ scan. Compared with CSE population, BSE individuals had different emphysema characteristic and severe airway remodeling, pectoralis muscle change and slow decline of pulmonary function. This suggests a different phenotype and progression.

\section{Acknowledgments}

We thank all the subjects who participated in this study and applied Chest Imaging Laboratory and Brigham and Women's Hospital for their great contribution to 3D slicer module. This study was supported by the National Key Research and
Development Program (2016YFC1304101), the Local Innovative and Research Teams Project of Guangdong Pearl River Talents Program (2017BT01S155), the National Natural Science Foundation of China (81970045), and Zhongnanshan Medical Foundation of Guangdong Province (ZNSA2020003, ZNSA-202012, ZNSA-2020013).

\section{Disclosure}

Authors have no competing interests. There are no financial relationships between our research team and any organizations that might have an interest in the submitted work. Other relationships or activities that might influence the submitted work were excluded throughout the study.

\section{References}

1. Halpin DMG, Criner GJ, Papi A, et al. Global initiative for the diagnosis, management, and prevention of chronic obstructive lung disease. The 2020 GOLD Science Committee Report on COVID-19 and Chronic Obstructive Pulmonary Disease. Am J Respir Crit Care Med. 2021;203(1):24-36. doi:10.1164/rccm.202009-3533SO

2. Pathak U, Gupta NC, Suri JC. Risk of COPD due to indoor air pollution from biomass cooking fuel: a systematic review and meta-analysis. Int $J$ Environ Health Res. 2020;30(1):75-88. doi:10.1080/09603123.2019.1575951

3. GBD 2016 Occupational Chronic Respiratory Risk Factors Collaborators; GBD 2016 occupational chronic respiratory risk factors collaborators. Global and regional burden of chronic respiratory disease in 2016 arising from non-infectious airborne occupational exposures: a systematic analysis for the Global Burden of Disease Study 2016. Occup Environ Med. 2020;77(3):142-150. doi:10.1136/ oemed-2019-106013

4. Deng Y, Gao Q, Yang D, et al. Association between biomass fuel use and risk of hypertension among Chinese older people: a cohort study. Environ Int. 2020;138:105620. doi:10.1016/j.envint.2020.105620

5. Imran M, Ozcatalbas O. Determinants of household cooking fuels and their impact on women's health in rural Pakistan. Environ Sci Pollut Res Int. 2020;27(19):23849-23861. doi:10.1007/s11356-02008701-8

6. Zhou Z, Dionisio KL, Arku RE, et al. Household and community poverty, biomass use, and air pollution in Accra, Ghana. Proc Natl Acad Sci U S A. 2011;108(27):11028-11033. doi:10.1073/ pnas. 1019183108

7. Siddharthan T, Grigsby MR, Goodman D, et al. Association between household air pollution exposure and chronic obstructive pulmonary disease outcomes in 13 low- and middle-income country settings. Am J Respir Crit Care Med. 2018;197(5):611-620. doi:10.1164/ recm.201709-18610C

8. Grajeda LM, Thompson LM, Arriaga W, et al. Effectiveness of gas and chimney biomass stoves for reducing household air pollution pregnancy exposure in guatemala: sociodemographic effect modifiers. Int J Environ Res Public Health. 2020;17(21):7723. doi:10.3390/ijerph17217723

9. Xu J, Bricker J, Fu X, et al. Design and development of smoking cessation apps based on smokers' and providers' perspectives in China: survey Study. JMIR Mhealth Uhealth. 2019;7(10):e12200. doi: 10.2196/12200

10. Luo T, Li MS, Williams D, et al. Using social media for smoking cessation interventions: a systematic review. Perspect Public Health. 2021;141(1):50-63. doi:10.1177/1757913920906845 
11. Naslund JA, Kim SJ, Aschbrenner KA, et al. Systematic review of social media interventions for smoking cessation. Addict Behav. 2017;73:81-93. doi:10.1016/j.addbeh.2017.05.002

12. Camp PG, Ramirez-Venegas A, Sansores RH, et al. COPD phenotypes in biomass smoke- versus tobacco smoke-exposed Mexican women. Eur Respir J. 2014;43(3):725-734. doi:10.1183/ 09031936.00206112

13. Zhao D, Zhou Y, Jiang C, Zhao Z, He F, Ran P. Small airway disease: a different phenotype of early stage COPD associated with biomass smoke exposure. Respirology. 2018;23(2):198-205. doi:10.1111/ resp. 13176

14. Tan WC, Sin DD, Bourbeau J, et al. Characteristics of COPD in never-smokers and ever-smokers in the general population: results from the CanCOLD study. Thorax. 2015;70(9):822-829. doi:10.1136/thoraxjnl-2015-206938

15. Díaz-Peña R, Silva RS, Hosgood HD 3rd, Agustí À, Olloquequi J. Specific miRNA Profile in Chronic Obstructive Pulmonary Disease Related to Biomass Smoke Exposure. Arch Bronconeumol (Engl Ed). 2021 Apr 15:S0300-2896(21)00123-X. English, Spanish. doi:10.1016/j.arbres.2021.03.020. Epub ahead of print.

16. Silva R, Oyarzún M, Olloquequi J. Pathogenic mechanisms in chronic obstructive pulmonary disease due to biomass smoke exposure. Arch Bronconeumol. 2015;51(6):285-292. doi:10.1016/j. arbres.2014.10.005

17. Liu S, Zhou Y, Liu S, et al. Association between exposure to ambient particulate matter and chronic obstructive pulmonary disease: results from a cross-sectional study in China. Thorax. 2017;72(9):788-795. doi:10.1136/thoraxjnl-2016-208910

18. Zhou Y, Zou Y, Li X, et al. Lung function and incidence of chronic obstructive pulmonary disease after improved cooking fuels and kitchen ventilation: a 9-year prospective cohort study. PLoS Med. 2014;11(3):e1001621. doi:10.1371/journal.pmed.1001621

19. Park J, Hobbs BD, Crapo JD, et al. Subtyping COPD by using visual and quantitative CT imaging features. Chest. 2020;157(1):47-60. doi:10.1016/j.chest.2019.06.015

20. Boueiz A, Chang Y, Cho MH, et al. Lobar emphysema distribution is associated with 5-year radiological disease progression. Chest. 2018;153(1):65-76. doi:10.1016/j.chest.2017.09.022

21. Takayanagi S, Kawata N, Tada Y, et al. Longitudinal changes in structural abnormalities using MDCT in COPD: do the CT measurements of airway wall thickness and small pulmonary vessels change in parallel with emphysematous progression? Int J Chron Obstruct Pulmon Dis. 2017;12:551-560. doi:10.2147/COPD.S121405

22. Matsuoka S, Washko GR, Yamashiro T, et al. Pulmonary hypertension and computed tomography measurement of small pulmonary vessels in severe emphysema. Am $J$ Respir Crit Care Med. 2010;181(3):218-225. doi:10.1164/rccm.200908-1189OC

23. Diaz AA, Martinez $\mathrm{CH}$, Harmouche R, et al. Pectoralis muscle area and mortality in smokers without airflow obstruction. Respir Res. 2018;19(1):62. doi:10.1186/s12931-018-0771-6
24. Suzuki Y, Yoshimura K, Enomoto Y, et al. Distinct profile and prognostic impact of body composition changes in idiopathic pulmonary fibrosis and idiopathic pleuroparenchymal fibroelastosis. Sci Rep. 2018;8(1):14074. doi:10.1038/s41598-018-32478-z

25. Lin H, Xiao D, Liu Z, Shi Q, Hajek P, Wang C. National survey of smoking cessation provision in China. Tob Induc Dis. 2019;17:25. doi: $10.18332 /$ tid $/ 104726$

26. Leone FT, Zhang Y, Evers-Casey S, et al. Initiating pharmacologic treatment in tobacco-dependent adults. An Official American Thoracic Society Clinical Practice Guideline. Am J Respir Crit Care Med. 2020;202(2):e5-e31. doi:10.1164/rccm.202005-1982ST

27. Wang Z, Chen X, Liu K, et al. Small pulmonary vascular alteration and acute exacerbations of COPD: quantitative computed tomography analysis. Int J Chron Obstruct Pulmon Dis. 2016;11:1965-1971. doi:10.2147/COPD.S112651

28. Cho YH, Lee SM, Seo JB, et al. Quantitative assessment of pulmonary vascular alterations in chronic obstructive lung disease: associations with pulmonary function test and survival in the KOLD cohort. Eur J Radiol. 2018;108:276-282. doi:10.1016/j.ejrad.2018.09.013

29. Juntarawijit C, Juntarawijit Y. Cooking with biomass fuel and cardiovascular disease: a cross-sectional study among rural villagers in Phitsanulok, Thailand. F1000Res. 2020;9:307. doi:10.12688/ f1000research.23457.2

30. Salvi SS, Brashier BB, Londhe J, et al. Phenotypic comparison between smoking and non-smoking chronic obstructive pulmonary disease. Respir Res. 2020;21(1):50. doi:10.1186/s12931-020-1310-9

31. Chan KH, Kurmi OP, Bennett DA, et al. Solid fuel use and risks of respiratory diseases. A cohort study of 280,000 Chinese never-smokers. Am J Respir Crit Care Med. 2019;199(3):352-361. doi:10.1164/rccm.201803-0432OC

32. Lim JU, Kim EK, Lim SY, et al. Mixed phenotype of emphysema and airway wall thickening is associated with frequent exacerbation in chronic obstructive pulmonary disease patients. Int J Chron Obstruct Pulmon Dis. 2019;14:3035-3042. doi:10.2147/COPD.S227377

33. McDonald ML, Diaz AA, Ross JC, et al. Quantitative computed tomography measures of pectoralis muscle area and disease severity in chronic obstructive pulmonary disease. A cross-sectional study. Ann Am Thorac Soc. 2014;11(3):326-334. doi:10.1513/ AnnalsATS.201307-2290C

34. Charbonnier JP, Pompe E, Moore C, et al. Airway wall thickening on CT: relation to smoking status and severity of COPD. Respir Med. 2019;146:36-41. doi:10.1016/j.rmed.2018.11.014

35. Teramoto S, Ishii M. Aging, the aging lung, and senile emphysema are different. Am J Respir Crit Care Med. 2007;175(2):197-198. doi:10.1164/ajrccm.175.2.197

36. Maselli DJ, Bhatt SP, Anzueto A, et al. Clinical epidemiology of COPD: insights From 10 years of the COPDGene Study. Chest. 2019;156(2):228-238. doi:10.1016/j.chest.2019.04.135

\section{Publish your work in this journal}

The International Journal of COPD is an international, peer-reviewed journal of therapeutics and pharmacology focusing on concise rapid reporting of clinical studies and reviews in COPD. Special focus is given to the pathophysiological processes underlying the disease, intervention programs, patient focused education, and self management protocols. This journal is indexed on PubMed Central, MedLine and CAS. The manuscript management system is completely online and includes a very quick and fair peer-review system, which is all easy to use. Visit http://www.dovepress.com/testimonials.php to read real quotes from published authors. 